\title{
ON THE CONVERGENCE OF SOME PROJECTION METHODS AND INEXACT NEWTON-LIKE ITERATIONS
}

\author{
IOANNIS K. ARGYROS
}

\begin{abstract}
We provide a general theorem for the convergence of some projection methods for inexact Newton-like iterations under Yamamoto-type assumptions. Our results extend and improve several situations already appeared in the literature.
\end{abstract}

\section{Introduction}

We consider the inexact Newton-like method

$$
x_{n+1}=x_{n}+y_{n^{\prime}} \quad P A\left(x_{n}\right) y_{n}=-\left(F\left(x_{n}\right)+G\left(x_{n}\right)\right)+r_{n^{\prime}} \quad n \geq 0
$$

for some $x_{0} \in U\left(x_{0}, R\right), R>0$, to approximate a solution $x^{*}$ of the equation

$$
F(x)+G(x)=0, \quad \text { in } \quad \bar{U}\left(x_{0}, R\right)
$$

Here $A(x), F, G$ denote operators defined on the closed ball $\bar{U}\left(x_{0}, R\right)$ with center $x_{0}$ and radius $R$, of a Banach space $E$ with values in a Banach space $\hat{E}$, whereas $r_{n}$ are suitable points in $\hat{E}$. The operator $A(x)(\cdot)$ is linear and approximates the Frechet derivative of $F$ at $x \in U\left(x_{0}, R\right) . P$ is a projection operator in $E$ such that $P^{2}=P$. We will assume that for any $x, y \in \bar{U}\left(x_{0}, r\right) \subseteq \bar{U}\left(x_{0}, R\right)$ with $0 \leq\|x-y\| \leq R-r$,

$$
\left\|P\left(F^{\prime}(x+t(x-y))-A(x)\right)\right\| \leq B_{1}\left(r,\left\|x-x_{0}\right\|+t\|y-x\|\right), t \in[0,1]
$$

and

$$
\|P(G(x)-G(y))\| \leq B_{2}(r,\|x-y\|)
$$

Received June 14, 1993.

1991 Mathematics Subject Classification. 47D15, 47H17, 65R20, 65J10.

Key words and phrases. Banach space, Newton-like method. 
The functions $B_{1}\left(r, r^{\prime}\right)$ and $B_{2}\left(r, r^{\prime}\right)$ defined on $[0, R] \times[0, R]$ and $[0, R] \times[0, R-r]$ are respectively nonnegative, continuous and non-decreasing functions of two variables. Moreover $B_{2}$ is linear in the second variable.

Note that the Newton method, the modified Newton method and the secant method are special cases of (1) with $A\left(x_{n}\right)=F^{\prime}\left(x_{n}\right), A\left(x_{n}\right)=F^{\prime}\left(x_{0}\right)$ and $A\left(x_{n}\right)=S\left(x_{n}, x_{n-1}\right)$ respectively (when $P=I$ the identity operator on $E$, or not).

If we take

$$
w\left(r^{\prime}\right)+c
$$

and

$$
e\left(r^{\prime}\right)
$$

where $w, e$ are nonnegative, nondecreasing functions on $[0, R-r]$, to be the right hand sides of (3) and (4) respectively, then we obtain conditions similar but not identical to the Zabrejko-Nguen-type assumptions considered by Chen and Yamamoto [2]. They provided sufficient conditions for the convergence of the sequence $\left\{x_{n}\right\}, n \geq 0$ generated by (1) to solution $x^{*}$ of equation (2), when $r_{n}=0, n \geq 0$ and $P=I$.

Moret [5] also studied (1), when $G=0$ and condition (5) is satisfied. Further work on this subject but for even more special cases than the ones considered by the above authors can be found in [1], [3], [4], [5], [6], [7], [8], [9], [10].

In this paper we will derive a criterion for controlling the residuals $r_{n}$ in such a way that the convergence of the sequence $\left\{x_{n}\right\}, n \geq 0$ to a solution $x^{*}$ of equation (2) is ensured.

We believe that conditions of the form (3)-(4) are useful not only because we can treat a wider range of problems than before, but it turns out that under natural assumptions we can find better error bounds on the distances $\left\|x_{n}-x^{*}\right\|, n \geq 0$.

The iterates $\left\{x_{n}\right\}$ generated by (1) when $P=I$ can rarely be computed in infinite dimensional spaces. It may be difficult or impossible to compute the inverses $A\left(x_{n}\right)$ at each step. It is easy to see however that the solution of equation (1) reduces to solving certain operator equations in the space $E_{p}$. If, moreover, $E_{p}$ is a finite dimensional space of dimension $N$, we obtain a system of linear algebraic equations of at most order $N$.

\section{Convergence Theorems.}

Throughout the paper the notation $\|\cdot\|$ will stand both for norms in $E$ (or in $\hat{E}$ ) and also for the induced operator norms $L(E, \hat{E})$, where $L(E, \hat{E})$ denotes the space of bounded linear operators from $E$ to $\hat{E}$.

We will need the following proposition.

Proposition. Let $a \geq 1, \sigma>0,0 \leq \mu<1,0 \leq \rho<R, s>0$ be real constants such that the equation

$$
\varphi(t):=a \sigma\left[\int_{0}^{t} B_{1}(R, \rho+\theta) d \theta+B_{2}(R, t)\right]-t(1-\mu)+s=0
$$


has solutions in the interval $[0, R)$ and let us denote by $x^{*}$ the least of them.

Let $v>0, \mu^{1} \geq 0$ such that

$$
v(1-\mu)-\left(1-\mu^{1}\right) \leq 0 .
$$

Then, for every $s^{1}$ satisfying

$$
0<s^{1} \leq v\left[\sigma\left(\int_{0}^{s} B_{1}(R, \rho+\theta) d \theta+B_{2}(R, s)\right)+s \mu\right]
$$

and for every $\rho^{1}$ such that

$$
0 \leq \rho^{1} \leq \rho+s
$$

the equation

$$
\varphi^{1}(t):=a v \sigma\left[\int_{0}^{t} B_{1}\left(R, \rho^{1}+\theta\right) d \theta+B_{2}(R, t)\right]-t\left(1-\mu^{1}\right)+s^{1}=0
$$

has nonnegative solutions and at least one of them, denoted by $t^{* *}$, lies in the interval $\left[s^{1}, t^{*}-s\right]$.

Proof. We first observe that since $\varphi\left(t^{*}\right)=0$ and $0 \leq \mu<1$, we obtain from (7) that $s \leq t^{*}$. We will show that

$$
\varphi^{1}\left(t^{*}-s\right) \leq 0 .
$$

Using (7)-(11), we obtain

$$
\begin{aligned}
\varphi^{1}\left(t^{*}-s\right)= & a v \sigma\left[\int_{0}^{t^{*}-s} B_{1}\left(R, \rho^{1}+\theta\right) d \theta+B_{2}\left(R, t^{*}-s\right)\right]-\left(t^{*}-s\right)\left(1-\mu^{1}\right)+s^{1} \\
\leq & v\left[a \sigma\left(\int_{s}^{t^{*}} B_{1}(R, \rho+\theta) d \theta+B_{2}\left(R, t^{*}\right)-B_{2}(R, s)\right)\right. \\
& \left.+\sigma\left(\int_{0}^{s} B_{1}(R, \rho+\theta) d \theta+B_{2}(R, s)\right)+s \mu-\frac{\left(t^{*}-s\right)}{v}\left(1-\mu^{1}\right)\right] \\
\leq & v\left[a \sigma\left(\int_{0}^{t^{*}} B_{1}(R, \rho+\theta) d \theta+B_{2}\left(R, t^{*}\right)\right)-t^{*}(1-\mu)+s\right. \\
& \left.+t^{*}(1-\mu)-s+s \mu-\frac{\left(t^{*}-s\right)}{v}\left(1-\mu^{1}\right)\right] \\
\leq & v\left(t^{*}-s\right)\left[(1-\mu)-\frac{\left(1-\mu^{1}\right)}{v}\right] \leq 0, \quad \text { by }(8) .
\end{aligned}
$$

Hence, $\varphi^{1}(t)$ has nonnegative real roots and for the least of them $t^{* *}$, it is

$$
s^{1} \leq t^{* *} \leq t^{*}-s .
$$

Moreover, from (11) we get $\mu^{1}<1$. 
That completes the proof of the proposition.

We can now prove the following result.

Theorem 1. Let $\left\{s_{n}\right\},\left\{\mu_{n}\right\},\left\{\sigma_{n}\right\}, n \geq 0$ be real sequences, with $s_{n}>0, \mu_{n} \geq$ $0, \sigma_{n}>0$. Let $\left\{\rho_{n}\right\}$ be a sequence on $[0, R)$, with $\rho_{0}=0$ and

$$
\rho_{n+1} \leq \sum_{j=0,1,2, \ldots, n} s_{j}, n \geq 0
$$

Suppose that $1-\mu_{0}>0$ and that, for a given constant $a \geq 1$, the function

$$
\varphi_{0}(t):=a \sigma_{0}\left[\int_{0}^{t} B_{1}\left(R, \rho_{0}+\theta\right) d \theta+B_{2}(R, t)\right]-t\left(1-\mu_{0}\right)+s_{0}
$$

has roots on $[0, R)$.

Assume that for every $n \geq 0$ the following conditions are satisfied

$$
\begin{gathered}
s_{n+1} \leq v_{n}\left[\sigma_{n}\left(\int_{0}^{s_{n}} B_{1}\left(R, \rho_{n}+\theta\right) d \theta+B_{2}\left(R, s_{n}\right)\right)+s_{n} \mu_{n}\right], \\
v_{n}\left(1-\mu_{n}\right)-\left(1-\mu_{n}\right) \leq 0,
\end{gathered}
$$

where $v_{n}=\frac{\sigma_{n+1}}{\sigma_{n}}$.

Then,

(a) for every $n \geq 0$, the equation

$$
\varphi_{n}(t):=a v_{n} \sigma_{n}\left[\int_{0}^{t} B_{1}\left(R, \rho_{n}+\theta\right) d \theta+B_{2}(R, t)\right]-t\left(1-\mu_{n}\right)+s_{n}
$$

has solutions in $[0, R)$ and, denoting by $t_{n}^{*}$ the least of them, we have

$$
\sum_{j=n, \ldots, \infty} s_{j} \leq t_{n}^{*}
$$

(b) Let $\left\{x_{n}\right\}, n \geq 0$ be a sequence in a Banach space such that $\left\|x_{n+1}-x_{n}\right\| \leq$ $s_{n}$. Then, it converges and denoting its limit by $x^{*}$, the error bounds

$$
\left\|x^{*}-x_{n}\right\| \leq t_{n}^{*}
$$

and

$$
\left\|x^{*}-x_{n+1}\right\| \leq t_{n}^{*}-s_{n}
$$

are true for all $n \geq 0$.

(c) If there exists $h_{0} \in[0, R)$ such that

$$
\varphi_{0}\left(h_{0}\right) \leq 0
$$


then $\varphi_{0}(t)$ has roots on $[0, R)$.

\section{Proof.}

(a) We use induction on $n$. Let us assume that for some $n \geq 0,1-\mu_{n}>0, \varphi_{n}(t)$ has roots on $[0, R)$ and $t_{n}^{*}$ is the least of them. This is true for $n=0$. Then, by (13), (15), (16) and the proposition, by setting $s=s_{n}, s^{1}=s_{n+1}, \mu=\mu_{n}, \mu^{1}=\mu_{n+1}$ and $v=v_{n}$, it follows that $t_{n+1}^{*}$ exists, with

$$
s_{n+1} \leq t_{n+1}^{*} \leq t_{n}^{*}-s_{n}
$$

and $1-\mu_{n+1}>0$.

That completes the induction and proves (a).

(b) This part follows easily from part (a).

(c) Using (21), we deduce immediately that $\varphi_{0}(t)$ has roots on $[0, R)$.

That completes the proof of theorem.

We can now prove the main result.

Theorem 2. Consider the method (1). Assume that for $s_{0}>0, \sigma_{0}>0,0 \leq$ $\mu_{0}<1$ and $a \geq 1,(21)$ is true. Then, the function $\varphi_{0}(t)$ defined by (14) has roots on $[0, R)$. Denote by $t_{0}^{*}$ the least of them and suppose that

$$
t_{0}^{*}<R_{0} \leq R
$$

Let $s_{n}>0, \mu_{n} \geq 0, \sigma_{n}>0, n \geq 0$ be such that $\lim \inf \sigma_{n}>0$ as $n \rightarrow \infty$ and condition (15) is true for all $n \geq 0$.

Assume that, for all $n \geq 0$,

$$
\left\|y_{n}\right\| \leq s_{n} \leq \sigma_{n}\left\|P\left(F\left(x_{n}\right)+G\left(x_{n}\right)\right)\right\|
$$

and

$$
\left\|P r_{n}\right\| \leq \frac{\mu_{n} s_{n}}{\sigma_{n}}
$$

Then the sequence $\left\{x_{n}\right\}, n \geq 0$ generated by (1) remains in $U\left(x_{0}, t_{0}^{*}\right)$ and converges to a solution $x^{*}$ of equation (2). Moreover, the error bounds (19) and (20) are true for all $n \geq 0$, where $t_{n}^{*}$ is the least root in $[0, R)$ of the function $\varphi_{n}(t)$ defined by (17), with $\rho_{n}=\left\|x_{n}-x_{0}\right\|, n \geq 0$.

Proof. The existence of $t_{0}^{*}$ is guaranteed by (21). Let us assume that $x_{n}, x_{n+1} \in$ $U\left(x_{0}, t_{0}^{*}\right)$. We will show that for every $n \geq 0$, condition (15) is true. Since $\left\|y_{0}\right\| \leq s_{0}$, this is true for $n=0$.

Using the identity

$$
\begin{aligned}
P\left(F\left(x_{n+1}\right)+G\left(x_{n+1}\right)\right) & =\int_{0}^{1} P\left[F^{\prime}\left(x_{n}+t\left(x_{n+1}-x_{n}\right)\right)-A\left(x_{n}\right)\right]\left(x_{n+1}-x_{n}\right) d t \\
& +P\left(G\left(x_{n+1}-G\left(x_{n}\right)\right)+P r_{n}\right.
\end{aligned}
$$


(3), (4), (23), (24), setting $\rho_{n}=\left\|x_{n}-x_{0}\right\|$ and by taking norms in the above identity we get

$s_{n+1} \leq \sigma_{n+1}\left\|P\left(F\left(x_{n+1}\right)+G\left(x_{n+1}\right)\right)\right\| \leq v_{n}\left[\sigma_{n}\left(\int_{0}^{s_{n}} B_{1}\left(R, \rho_{n}+\theta\right) d \theta+B_{2}\left(R, s_{n}\right)\right)+s_{n} \mu_{n}\right]$ which shows (15) for all $n \geq 0$.

The hypothesis (b) of Theorem 1 can now easily be verified by induction and thus, by(18) and (23), the sequence $\left\{x_{n}\right\}, n \geq 0$ remains in $U\left(x_{0}, t_{0}^{*}\right)$, converges to $x^{*}$ and (19) and (20) hold. Finally, from the inequality

$$
\left\|P\left(F\left(x_{n}\right)+G\left(x_{n}\right)\right)\right\| \leq\left\|P\left(A\left(x_{n}\right)-F^{\prime}\left(x_{0}\right)\right)\right\|\left\|y_{n}\right\|+\left\|P F^{\prime}\left(x_{0}\right)\right\|\left\|y_{n}\right\|+\left\|P r_{n}\right\|,
$$

(3), (24) and the continuity of $F$ and $G$, as $\liminf \sigma_{n}>0$ and $s_{n} \rightarrow 0$, as $n \rightarrow \infty$ it follows that $P\left(F\left(x^{*}\right)+G\left(x^{*}\right)\right)=0$. Hence $F\left(x^{*}\right)+G\left(x^{*}\right)=0$.

That completes the proof of the theorem.

\section{Remark.}

(a) In the special case when $B_{1}$ and $B_{2}$ are given (5) and (6) respectively, then our results can be reduced to the ones obtained by Moret [5, p.359] (when $G=0$ and $P=I)$.

(b) Let $G=0, P=I, A(x)=F^{\prime}(x)$ and define the functions $\bar{\varphi}_{0}(t), \bar{\varphi}_{n}(t)$ by

$$
\begin{gathered}
\bar{\varphi}_{0}(t)=a \sigma_{0} \int_{0}^{t}(t-\theta) k(\theta) d \theta-t\left(1-\mu_{0}\right)+s_{0}, \\
\bar{\varphi}_{n}(t)=a v_{n} \sigma_{n} \int_{0}^{t}(t-\theta) k\left(\rho_{n}+\theta\right) d \theta-t\left(1-\mu_{n}\right)+s_{n},
\end{gathered}
$$

where $k$ is a nondecreasing function on $[0, R]$ such that

$$
\left\|F^{\prime}(x)-F^{\prime}(y)\right\| \leq k(r)\|x-y\|, \quad x, y \in \bar{U}\left(x_{0}, r\right) \quad\left(r<R_{0}\right) .
$$

Assume that $B_{1}$ can be chosen in such a way that

$$
\varphi_{n}(t) \leq \bar{\varphi}_{n}(t), n \geq 0 .
$$

The under the hypotheses of Theorem 2 above and Proposition 1 in [5, p. 359], using (25) we can show

$$
\left\|x^{*}-x_{n}\right\| \leq t_{n}^{*} \leq m_{n}^{*}, n \geq 0
$$

and

$$
\left\|x^{*}-x_{n+1}\right\| \leq t_{n}^{*}-s_{n} \leq m_{n}^{*}-s_{n}, n \geq 0
$$

where by $m_{n^{\prime}}^{*}$ we denote the least solutions of the equations

$$
\bar{\varphi}_{n}(t)=0, n \geq 0 \quad \text { in } \quad[0, R) .
$$




\section{References}

[1] P.T. Brown, "A local convergence theory for inexact-Newton finite-difference projection methods," SIAM J. Numer. Anal., 24, (1987), 407-437.

[2] X. Chen, T. Yamamoto, "Convergence domains of certain iterative methods for solving nonlinear equations," Numer. Funct. Anal. and Optimiz., 10, (1 and 2), (1989), 37-48.

[3] R.S. Dembo, S.C. Eisenhart, and T. Steihaug, "Inexact Newton methods," SIAM J. Numer. Anal., 19, (1982), 400-408.

[4] M.A. Krasnosel'skii, and Y.B. Rutickii, "Some approximate methods of solving nonlinear equations based on linearization," Soviet Math. Dokl., 2, (1961), 1542-1546.

[5] I. Moret, "A Kantorovich-type theorem for inexact Newton methods," Numer. Funct. Anal. and Optimiz., 10, (3 and 4), (1989), 351-365.

[6] F.A. Potra, and V. Ptàk, Nondiscrete induction and iterative processes., Pitman Advanced Publishing Program, London, 1984.

[7] A.H. Sherman, "On Newton-iterative methods for the solution of systems of equations," SIAM J. Numer. Anal., 15, (1978), 755-771.

[8] P.P. Zabrejko, and D.F. Nguen, "The majorant method in the theory of Newton-Kantorovich approximations and the Ptàk error estimates," Numer. Funct. Anal. and Optimiz., 9, (1987), 671684.

[9] T. Yamamoto, "A note on a posterior error bound of Zabrejko and Nguen for Zincenko's iteration," Numer. Funct. Anal. and Optimiz., 9, (1987), 987-994.

[10] T.J. Ypma, "Local convergence of inexact Newton methods," SIAM J. Numer. Anal., 21, (1984), 583-590.

Cameron University, Department of Mathematics, Lawton, OK 73505-6377, U. S. A. 